\title{
LA MECCANICA DELLE MACCHINE NELL'INNOVAZIONE DEI PRODOTTI E DEI PROCESSI
}

\author{
MASSIMO SORLI (*)
}

SunTO. - Il testo riassume la presentazione svolta durante il simposio MECCANICA TEORICA E APPLICATA del 13 ottobre 2016. La relazione, dopo una breve introduzione riguardante sinteticamente anche i cenni storici, identifica e presenta le diverse aree che compongono disciplinarmente la Meccanica delle Macchine, esponendo in una forma sostanzialmente matriciale le tecnologie abilitanti da un lato e i domini applicativi dall'altro, a cui queste tipicamente si applicano. Viene quindi presentata una sintesi delle attività attualmente in corso nelle diverse sedi degli Atenei italiani, dalla quale si può evidenziare la metodologia degli studi affrontati, fortemente rivolta a un approccio sistemistico unificante, mediante le tecniche proprie della meccanica teorica, applicata e sperimentale, con attenzione alla sostenibilità ambientale ed energetica, e significativamente connessa da un lato con lo stato dell'arte della ricerca internazionale, e dall'altro con la realtà industriale e produttiva del paese. Vengono infine citati alcuni settori della Meccanica delle Macchine che a parere dello scrivente necessitano di essere investigati ulteriormente, nel campo per esempio delle trasmissioni e degli argani di attuazione. A tale proposito sono delineate alcune sfide tecnologiche che evidenziano il contributo alla innovazione dei processi e dei prodotti, quali i modelli di prognostica applicati a servosistemi nei comandi di volo primari in applicazioni aeronautiche, sfide che necessitano di risalire alle interazioni input-output della meccanica di base, senza le quali risulta impossibile potere predire l'evoluzione delle degradazioni in tali sistemi di attuazione, e tanto meno arrivare a determinare la vita residua di un dispositivo avente funzionalità meccanica.

$$
* * *
$$

ABSTRACT. - The present report highlights the current and future role of techniques and methodologies of the Mechanics of Machines, both in the design of devices and systems, and in the university training courses. The underlying theme of the presentation lays in the interpretation of the physical phenomenon which oversees the operation of the machines. This is the foundation allowing to define an input-output interaction

(*) Dipartimento di Ingegneria Meccanica e Aerospaziale. Politecnico di Torino, Italia. E-mail: massimo.sorli@polito.it 
between the physical quantities operating on the machine. The cause-effect relation offers the possibility to determine a set of analytical relations for the prediction of the operation of the machine and to simulate theoretical and / or numerical trends in time or frequency domain of the significant mechanical quantities. It is evident the magnitude of the physical phenomena that arise in the operation of a machine, resulting in a broad variety of related Mechanics of Machines topics: from the contact between bodies analysis to tribological aspects, from body geometry to kinematics, from the rigid to deformable body dynamics, from the interaction between mechanical bodies to manmachine interaction, from the kinematic and dynamic behavior of a mechanical system to its interface with the actuators, sensing and control, just to name some of them. It should also be considered that the interpretation of the physical phenomenon of organs of machines has to be supported by significant experimental campaigns, specifically reproduced in laboratory, or related to data from real applications in the different application domains. The evolution of Mechanics of Machines proved in the years to be able to respond to these two interacting and converging questions: on the one hand the need to identify analytical relations, possibly not based on sole mappings of data, but rather on representative analytical relations of physical quantities, and on the other hand, the need, even at the university level, to conduct appropriate laboratory test campaigns related to the real field operation of the machine. With reference to the first objective, the need to determine algorithms, typically non-linear, and the consequent simulations setups, has resulted in the passage of the Machine Mechanics from a theoretical subject, to a subject with strong computational valences creating tools for the prediction of the behavior of devices and systems, in relation to their diagnosis and health state. The second objective has required the achievement of competence also in the field of the test rigs, of sensing and measuring / data acquisition systems. The paper deals with the identification and the presentation of the different areas related to Machine Mechanics, exposing in a matrix the enabling technologies on the one hand and the application domains to which they apply in the other hand. The enabling technologies traditionally belong to the topics of kinematics, statics, dynamics (linear and nonlinear), to the interactions with the environment (force fields, interactions with fluids) and between surfaces (lubrication), control, automation and system identification, as well as to the study and identification of vibratory phenomena, vibro-acoustic and tribological ones, mechatronics, fluid-structure interactions, monitoring, diagnostics and prognostics of mechanical systems, fluid automation and robotics, fluidics and microfluidics, to the implementation of pneumatic, hydraulic, electric and non-conventional technologies, to environmentally friendly and renewable energy systems. The application domains relate to the mechanical systems, such as driving and operating machinery, mechanical devices, mechanisms, transmissions and drives, automatic and robotic, vehicles on road, rail, fixed wing and rotorcrafts, transportation and lifting systems, systems for the production of energy, the biomechanical systems. A summary of the ongoing activities in the different research groups of the Italian Universities is then presented, from which you can also highlight the methodology of the studies addressed, strongly aimed at a unifying approach through the use of fundamental methods of theoretical applied and experimental mechanics, with attention to environmental and energy sustainability, and significantly connected on one side with the state of international research, and on the other with the industrial and manufacturing reality of the country. At the end of the paper sectors of Machine Mechanics that in the opinion of the writer need to be investigated further are discussed. Some technological challenges, such as 
prognostic models applied to servo systems in primary flight controls for aircraft applications, are outlined. The state of the art in that domain highlights the contribution to the innovation of processes and products, challenge that need to go back to the inputoutput interactions at the base-mechanics layer. Without those aspects it is impossible to be able to predict the evolution of degradation in the actuation systems, and to determine the remaining life of a mechanical device.

\section{INTRODUZIONE}

La relazione è focalizzata a evidenziare il ruolo attuale e futuro delle conoscenze e delle metodologie proprie della Meccanica delle Macchine (MdM nel testo a seguire), sia nella progettazione dei dispositivi e dei sistemi, sia nei percorsi di formazione universitaria.

Il filo conduttore della presentazione è il seguente: l'interpretazione del fenomeno fisico che sovrintende al funzionamento delle macchine è il fondamento che permette di definire una interazione input-output fra le grandezze fisiche operanti sulla macchina. Questa relazione di causa-effetto offre la possibilità di determinare una serie di relazioni analitiche utili alla predizione del funzionamento della macchina, atte cioè a simulare in via teorica e/o numerica gli andamenti nel dominio del tempo o della frequenza delle grandezze meccaniche significative.

Risulta evidente l'ampiezza dei fenomeni fisici che si determinano nel funzionamento di una macchina, e conseguente è la complessità degli argomenti propri della MdM: dalle modalità di contatto fra i corpi agli aspetti tribologici, da quelli connessi con la geometria degli organi a quelli cinematici, dalla dinamica di corpo rigido a quella di corpo deformabile, dalla interazione fra corpi meccanici alla interazione uomo-macchina, dal comportamento cinematico e dinamico di un sistema meccanico al suo interfacciamento con gli organi di attuazione, sensorizzazione e controllo, solo per citarne alcuni.

Occorre altresì considerare che l'interpretazione del fenomeno fisico fra organi di macchine non può che essere avvalorato da significative campagne sperimentali, siano esse di laboratorio, o siano correlate con dati provenienti dal campo, ossia da applicazioni reali presenti nei differenti domini applicativi [1], [2].

L'evoluzione della Meccanica delle Macchine si è determinata negli anni per potere rispondere a queste due domande interagenti e convergenti: da un lato la necessità di individuare relazioni analitiche, possibilmente non basate su sole mappature di dati, ma bensì su rela- 
zioni funzionali stazionarie e dinamiche, rappresentative di grandezze fisiche; dall'altro la necessità, anche a livello universitario, di svolgere opportune campagne di prova di laboratorio, che fossero significative del reale funzionamento sul campo della macchina.

Con riferimento al primo obiettivo, la necessità si determinare algoritmi di calcolo, tipicamente non lineari, e la conseguente conduzione di simulazioni, ha comportato il passaggio della Meccanica delle Macchine da una materia originariamente prevalentemente teorica, ad una materia avente spiccate valenze computazionali, fino agli attuali strumenti atti alla predizione del comportamento dei dispositivi e dei sistemi durante la loro vita operativa, relativamente alla diagnostica e allo stato di salute degli stessi.

Il secondo obiettivo ha richiesto il raggiungimento di competenze anche nel settore dei banchi di prova [3], dei sistemi di sensorizzazione, di misura e acquisizione dati, in definitiva ha richiesto la crescita di conoscenza nella realizzazione di setup di prova.

La sintesi dei due obiettivi sopra indicati è a parere dello scrivente fondamentale, al fine di potere ottenere algoritmi che non solo identifichino il comportamento della macchina in una condizione di funzionamento, ma siano validati anche in condizioni di funzionamento vicine, ossia al variare dei parametri, in altri termini siano in grado di predire il comportamento statico e dinamico del sistema.

\section{LA COLLOCAZIONE MINISTERIALE DELLA MdM}

Laddove e allorquando si tratti di un sistema che ha funzionalità meccanica, ossia di un sistema che debba regolare azioni per determinare leggi e grandezze cinematiche, pur in presenza di una sempre maggiore integrazione e trasversalità delle tecnologie, gli organi delle macchine giocano e giocheranno un ruolo fondamentale e indispensabile. Ciò premesso, il contributo della MdM deve essere perseguito attraverso una continua innovazione e una sempre più accurata e efficace interpretazione dei fenomeni che stanno alla base del funzionamento dei sistemi meccanici.

La MdM trova nella Enciclopedia Treccani una sua esplicitazione all'interno della Meccanica Applicata, come espressione della Meccanica Applicata alle Machine (MAM). La Meccanica Applicata (MA) è definita come: Complesso delle discipline in cui trovano applicazione i principi e 
i metodi della meccanica razionale. È possibile distinguere la MA in MA alle Costruzioni, MA ai Fluidi, MA alle Macchine. Per quanto riguarda quest'ultima viene indicato quanto segue:

La Meccanica Applicata alle Macchine concerne gli elementi delle macchine, sia riguardo alla loro capacità di trasmettere moto sia riguardo a quello di trasmettere forze. Se lo studio è limitato all' individuazione del moto consentito ai diversi organi delle macchine in conseguenza dei vincoli, sia esterni che interni, tale studio è essenzialmente cinematico.

I contenuti della MdM si ritrovano pienamente nella declaratoria del settore concorsuale SC 09/A2 coincidente con il Settore Scientifico Disciplinare SSD ING-IND/13 - MECCANICA APPLICATA ALLE MACCHINE (Decreto Ministeriale 30 ottobre 2015 n. 855):

Il settore si interessa dell' attività scientifica e didattico-formativa nel campo della Meccanica Applicata alle Macchine. Il settore comprende gli aspetti culturali, scientifici e professionali inerenti lo studio dei sistemi meccanici, delle macchine e dei loro componenti e delle strutture: lo studio viene affrontato, con un approccio sistemistico unificante, mediante le metodologie proprie della meccanica teorica, applicata e sperimentale, sfociando nell' applicazione tecnologica e industriale, con attenzione alla sostenibilità ambientale ed energetica. La tipologia dei sistemi meccanici considerati è del tutto generale: macchine motrici ed operatrici, dispositivi meccanici, meccanismi, trasmissioni ed azionamenti, macchine automatiche e robot, veicoli, sistemi di trasporto e sollevamento, sistemi per la produzione di energia, sistemi biomeccanici, componenti e sistemi su scala micro/nano. Sono sviluppati metodi teorici e sperimentali ed applicazioni relativi all'analisi del comportamento meccanico, alla sintesi, e alla progettazione, in particolare funzionale, delle macchine e dei sistemi meccanici, tramite lo studio della cinematica, della statica, della dinamica, lineare e non lineare, delle interazioni con l'ambiente (campi di forze, interazioni con i fluidi) e fra superfici materiali (lubrificazione), del controllo dell'automazione e dell' identificazione. L'implementazione tramite sistemi hardware e software analogici e digitali dei metodi sviluppati costituisce parte integrante del sapere del settore. Come ulteriore risposta a esigenze di progettazione, sviluppo e realizzazione di sistemi e componenti innovativi, sono anche studiati: i fenomeni vibratori, vibroacustici e tribologici, il controllo dei sistemi meccanici, la meccatronica, le interazioni fluidostrutture, il monitoraggio, la diagnostica e la prognostica di sistemi meccanici, l'automazione a fluido e la robotica, la fluidica e la microfluidica, i sistemi ecocompatibili e le energie rinnovabili. Il settore approfondisce inoltre le problematiche inerenti i sistemi di attuazione pneumatici, idraulici, elettrici e basati su tecnologie non convenzionali (ad esempio, materiali intelligenti) che ormai fanno parte integrante, insieme ai sistemi di controllo, delle macchine, dei sistemi meccatronici e di molte strutture. Forti interrelazioni si attuano con le metodologie e gli algoritmi sviluppati 
nel settore del disegno, con i metodi dell'ingegneria industriale, della progettazione dimensionale e della costruzione delle macchine, della fluidodinamica, della bioingegneria, delle scienze motorie, della chirurgia ortopedica e protesica, delle metodologie per riabilitazione e assistenza ed infine con la interpretazione e la analisi di macchine di interesse storico.

La collocazione del SC 09/A2 all'interno del Macrosettore 09/A Ingegneria Meccanica, Aerospaziale e Navale è mostrato nella tabella di Fig. 1.

\begin{tabular}{|c|c|c|}
\hline \begin{tabular}{|l} 
MACROSETTORE \\
Codice e Denominazione
\end{tabular} & $\begin{array}{l}\text { SETTORE CONCORSUALE } \\
\text { Codice e Denominazione }\end{array}$ & $\begin{array}{l}\text { SETTORE SCIENTIFICO-DISCIPLINARE } \\
\text { Codice e Denominazione }\end{array}$ \\
\hline \multicolumn{3}{|l|}{\begin{tabular}{|l|} 
09/A-INGEGGERIA \\
MECCANICA, AEROSPAZIALE \\
E NAVALE
\end{tabular}} \\
\hline & 09/A1 - INGEGNERIA AERONAUTICA, AEROSPAZIALE E NAVALE & $\begin{array}{l}\text { ING-IND/01 - ARCHITETTURA NAVALE } \\
\text { ING-IND/02 - COSTRUZIONI E IMPIANTI NAVALI E } \\
\text { MARINI } \\
\text { ING-IND/03 - MECCANICA DEL VOLO } \\
\text { ING-IND/04 - COSTRUZIONI E STRUTTURE } \\
\text { AEROSAZIALI } \\
\text { ING-IND/O6 - FLUIDODINAMICA } \\
\text { ING-IND/05 - IMPIANTE SISTEMI AEROSPAZIALI } \\
\text { ING-IND/07 - PROPULSISINE AEROSPAZIALE. }\end{array}$ \\
\hline & 09/A2 - MECCANICA APPLLICATA ALLLE MACCHINE & $\begin{array}{l}\text { ING-IND/13-MECCANICA APPLICATA ALLE. } \\
\text { MACCHNE }\end{array}$ \\
\hline & $\begin{array}{l}\text { 09/A3 - PROGETTAZIONE INDUSTRIALE, COSTRUZIONI MECCANICHE } \\
\text { E METALLURGIA }\end{array}$ & $\begin{array}{l}\text { ING-IND/14 - PROGETTAZIONE MECCANICA E } \\
\text { COSTRUZIONE DI MACCHINE } \\
\text { ING-IND/IS - DISEGNO E METODI DELLL'INGEGNERIA } \\
\text { INDUSTRIALE } \\
\text { ING-IND/2I - METALLURGIA }\end{array}$ \\
\hline
\end{tabular}

Fig. 1. Collocazione del SC 09/A2 all'interno del Macrosettore 09/A.

L'attività di ricerca e formativa della accademia in Italia, nel campo della MdM, si identifica pienamente nel SSD ING_IND/13 Meccanica Applicata alle Macchine; i colleghi afferenti a tale SSD si coordinano a livello nazionale nel Gruppo di Meccanica Applicata (GMA).

\section{CENNI STORICI}

Arduo e velleitario è potere riassumere in poche righe il percorso della MdM nel tempo, dai primi utensili preistorici, ai dispositivi e alle macchine dei Greci e dei Romani, basati su leve, cunei, ruote, pulegge, viti, ... fino alla evoluzione della scienza del tardo medioevo. Sembra di potere affermare che prima del 1500 non ci fosse una piena consapevolezza e una completa interpretazione delle motivazioni teoriche secondo le quali le macchine funzionassero. Nell'intorno del XVI secolo, [4] e [5], iniziò a emergere lo studio del progetto dei Meccanismi e della teoria della Meccanica delle Macchine. Nel seguito sono accennati alcuni riferimenti miliari: 
- Leonardo da Vinci, 1452-1519: moto di un corpo su piano inclinato, definizione di forza e di risultante di forze.

- Galileo Galilei, 1564-1642: caduta dei gravi, centro di gravità, accelerazione di gravità.

- Christiaan Huygens, 1629-1695: urti, principio di inerzia.

- Isaac Newton, 1642-1727: massa, quantità di moto, forza d'inerzia, regola del parallelogramma, legge di gravitazione universale. Pubblica nel 1687 Philosophiae naturalis principia matematica, testo di base sulla meccanica classica. Sua la celebre affermazione, "Hypotheses non fingo", in base alla quale egli si riprometteva di rifiutare qualsiasi spiegazione della natura che prescindesse da una solida verifica sperimentale.

Fino alla fine del Rinascimento le attività principali erano rivolte alla progettazione di fortificazioni, ponti, mulini, opere idrauliche e macchine militari. Gli organi di macchine principalmente impiegati erano la leva, la ruota, la puleggia, il cuneo, la vite, essendo la potenza motrice prevalentemente umana o animale.

Significativo l'anno 1712, data di invenzione della macchina a vapore, che determinò: maggiore capacità di pompaggio di acqua dalle miniere, conseguente incremento produttivo, necessità di nuovi dispositivi, sistemi, macchine. Si ritiene che in tale periodo inizi l'era dell'ingegneria meccanica.

Nella seconda metà del '700 si determina la Prima Rivoluzione Industriale, praticamente sinonimo di Meccanizzazione, si accelerano una serie di processi che conducono allo sviluppo della ingegneria meccanica, esempio tipico si evidenzia nelle innovazioni delle macchine dell'industria tessile.

Alla fine del XVIII secolo viene fondata a Parigi l'École Polytechnique (1794), che faceva seguito all'École du Genie Militaire (1748). Si iniziano a classificare le macchine e i meccanismi, in relazione alla tipologia di movimento di ingresso e di uscita, si studia la cinematica dei meccanismi. Inizia in tale epoca la Formazione di Ingegneri, che si concretizza poi nel XIX secolo.

Fra la fine dell'‘ 800 e l'inizio del '900 si ha la seconda Rivoluzione Industriale. Essa è caratterizzata dalla comparsa della elettricità, del motore a combustione interna, dell'acciaio, di nuove leghe, delle tecnologie di comunicazione (telegrafo e radio), con conseguente aumento della produttività, e sempre più marcato ruolo fondamentale degli orga- 
ni di macchine.

Nella prima metà ' 900 si riscontra una produzione industriale di larga scala, macchine veloci, fabbriche organizzate, specialmente in Europa e negli Stati Uniti. Si evidenzia la necessità di passare dalla teoria delle macchine e dei meccanismi alla realizzazione efficace di macchine che svolgano determinate funzioni. Il periodo bellico offre applicazioni di tale progresso tecnologico, purtroppo non esemplari.

Nei successivi anni '50-'80 si ha l'esplosione della materia della MdM in diversi settori, nasce l'automazione, si passa da meccanismi piani a meccanismi sferici e spaziali, si consolidano le strutture a più g.d.l.

Gli ultimi 30 anni vedono una sempre maggiore integrazione fra la meccanica, l'elettronica e l'informatica, si ha una evoluzione verso macchine controllate e sistemi meccatronici; si riscontrano altresì richieste della società moderna per ausili per la salute, nel campo della automazione robotica assistita, della biomeccanica, della riabilitazione.

Oggi Meccanica teorica - Sperimentazione - Modellazione Simulazione numerica sono parti integranti della risposta alla domanda di innovazione che è in essere nel settore della MdM, da un lato con una sempre maggiore integrazione con altre discipline e, dall'altro e allo stesso tempo, con una sempre maggiore richiesta di più approfondita interpretazione dei fenomeni fisici che si determinano all'interno degli organi di macchine.

La MdM è una disciplina attuale e con significative e indubbie prospettive future. E' indubbio che l'essere umano opera e interagisce con l'ambiente in cui vive attraverso azioni che hanno natura meccanica (forze, coppie). I dispositivi meccanici, in generale gli organi di trasmissione, i sistemi di asservimento, giocano un ruolo fondamentale nell'ottimizzazione di tali azioni, e possono contribuire a ridurre lo sforzo applicato, possono assistere o addirittura sostituirsi all'essere umano, peraltro con tutte le implicazioni che ne derivano.

\section{Matrice DOMini APPliCATIVI/TECNOLOGIE ABILITANTI DELLA MDM}

I domini applicativi in cui opera la MdM sono quelli tipici in cui si collocano i sistemi meccanici e meccatronici. Essi possono essere sin- 
tetizzati nel seguente elenco:

- le macchine motrici ed operatrici, i dispositivi meccanici, i meccanismi, gli organi di trasmissione e gli azionamenti;

- le macchine automatiche e robotiche, l'automazione manifatturiera;

- i sistemi biomeccanici e biomedici, gli ausili per disabili;

- i sistemi per la mobilità, i veicoli su gomma, su rotaia, i velivoli ad ala fissa e rotante, lo spazio, i sistemi marini e sottomarini, i sistemi a guida autonoma;

- i sistemi di trasporto e sollevamento;

- i sistemi meccatronici per la trasformazione di energia, in particolare per energie rinnovabili (vento, sole, marino, idrico).

Le tecnologie abilitanti si sono progressivamente aggiornate negli anni recenti, in relazione alla evoluzione della tecnologia, passando da tematiche maggiormente teoriche verso analisi e sviluppi rivolti alle reali richieste implementative della MdM. Originarie aree della MdM erano l'Analisi e la Sintesi dei Meccanismi, la Cinematica teorica, la Meccanica dei corpi rigidi, la Meccanica dei macchinari, la Dinamica dei rotori, il progetto di Sistemi meccanici per l'automazione, la Regolazione dei sistemi meccanici, la Biomeccanica, la Storia della Teoria delle macchine e dei meccanismi.

Pur rimanendo attuali tali originarie tematiche, le attuali tecnologie abilitanti della MdM si sono evolute e oggi comprendono:

- Cinematica computazionale, dinamica lineare e non lineare, dinamica multibody;

- Identificazione dei fenomeni vibratori e vibroacustici;

- Tribologia e identificazione dei fenomeni tribologici, interazione fra superfici materiali (lubrificazione) e fluido-strutture;

- Robotica, di servizio, collaborativa, robotica mobile, robotica spaziale;

- Automazione a fluido, fluidica e microfluidica;

- Meccanismi e microsistemi;

- Meccatronica, controllo e regolazione dei sistemi meccanici;

- Biomeccanica, interfaccia uomo-macchina, dispositivi biomeccanici e medicali, ergonomia;

- Monitoraggio, Diagnostica e Prognostica dei sistemi meccanici;

- Sistemi di attuazione pneumatici, idraulici, elettrici, servosistemi, basati anche su tecnologie non convenzionali;

- Sistemi ecocompatibili, Meccanica sostenibile; 
- Dispositivi meccatronici per sistemi di energie rinnovabili.

I comparti di applicazione della MdM possono essere raggruppati nel modo seguente:

- Automotive, Aerospazio, Ferroviario, Navale

- Food

- Energia rinnovabile

- Macchine agricole

- Manifatturiero, Sistemi avanzati di produzione

- Infrastrutture civili

- Riabilitazione, medicina, salute

- Tessile

I metodi impiegati da ricercatori della $\mathrm{MdM}$, con un approccio multidisciplinare, inclusivo anche di altre aree della ingegneria, non solo meccanica, fanno riferimento ai seguenti approcci:

- Interpretazione del fenomeno fisico, Modellazione analitica;

- Simulazione numerica;

- Sperimentazione di laboratorio, sperimentazione sul campo, realizzazione di prototipi;

- Analisi prestazionale, identificazione e valutazione delle performance;

- Analisi del ciclo di vita, interpretazione dello stato di salute;

- Valutazione delle meriti economici e commerciali di un dispositivo;

- Valutazione della ricaduta sociale, interazione con l'essere umano, sostenibilità e fruibilità.

\section{ATtUALI E PREVALENTI CAMPI DI RICERCA DELLA MDM}

Come si è detto nei paragrafi precedenti, è praticamente impossibile trovare un settore applicativo della tecnologia nel quale non sia coinvolto un sistema meccanico/meccatronico di cui è necessario studiarne l'equilibrio statico o dinamico, o non sia necessario valutarne l'efficienza energetica, o non sia opportuno ridurne dimensioni/masse o limitarne gli effetti inerziali, o ancora non sia richiesto di ridurre la trasmissione di vibrazioni e rumore.

Quanto indicato a seguire vuole quindi essere solo una traccia delle attività di ricerca [6], che sono in essere nel corso del 2016 presso 
le diverse sedi accademiche degli Atenei italiani nel settore della MdM (Figs. 2-20), senza alcuna valenza esaustiva e tanto meno senza alcun obiettivo di accreditare le metodologie e i risultati conseguiti. Per le considerazioni di metodo e di obiettivo si rimanda quindi ai siti web dei singoli colleghi e gruppi di ricerca, e a quello del Gruppo di Meccanica Applicata (GMA).

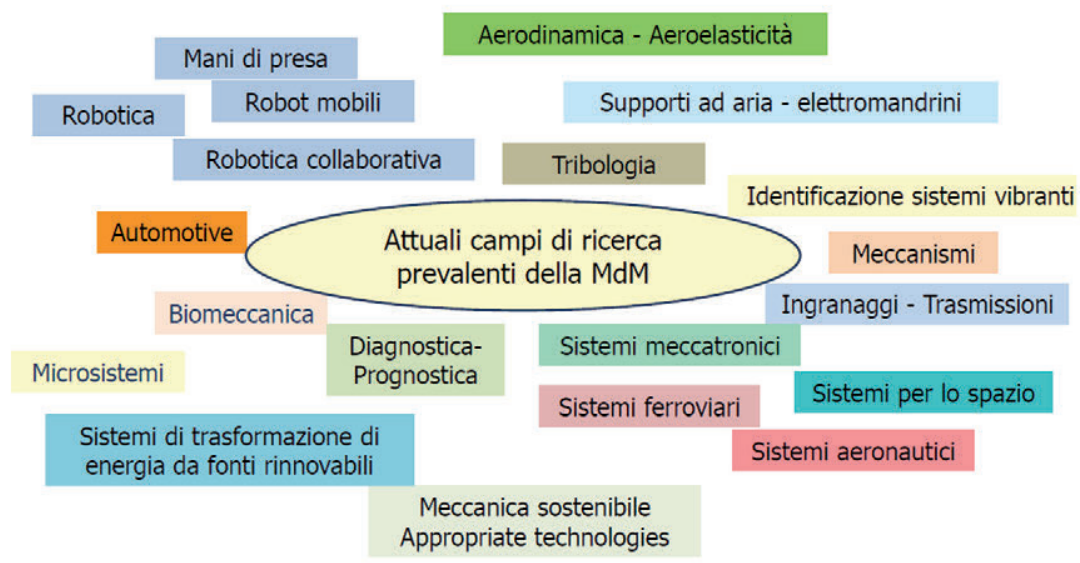

Fig. 2. Campi di ricerca della $M d M$ negli Atenei italiani 




Fig. 3. Ricerche nel campo Automotive. 


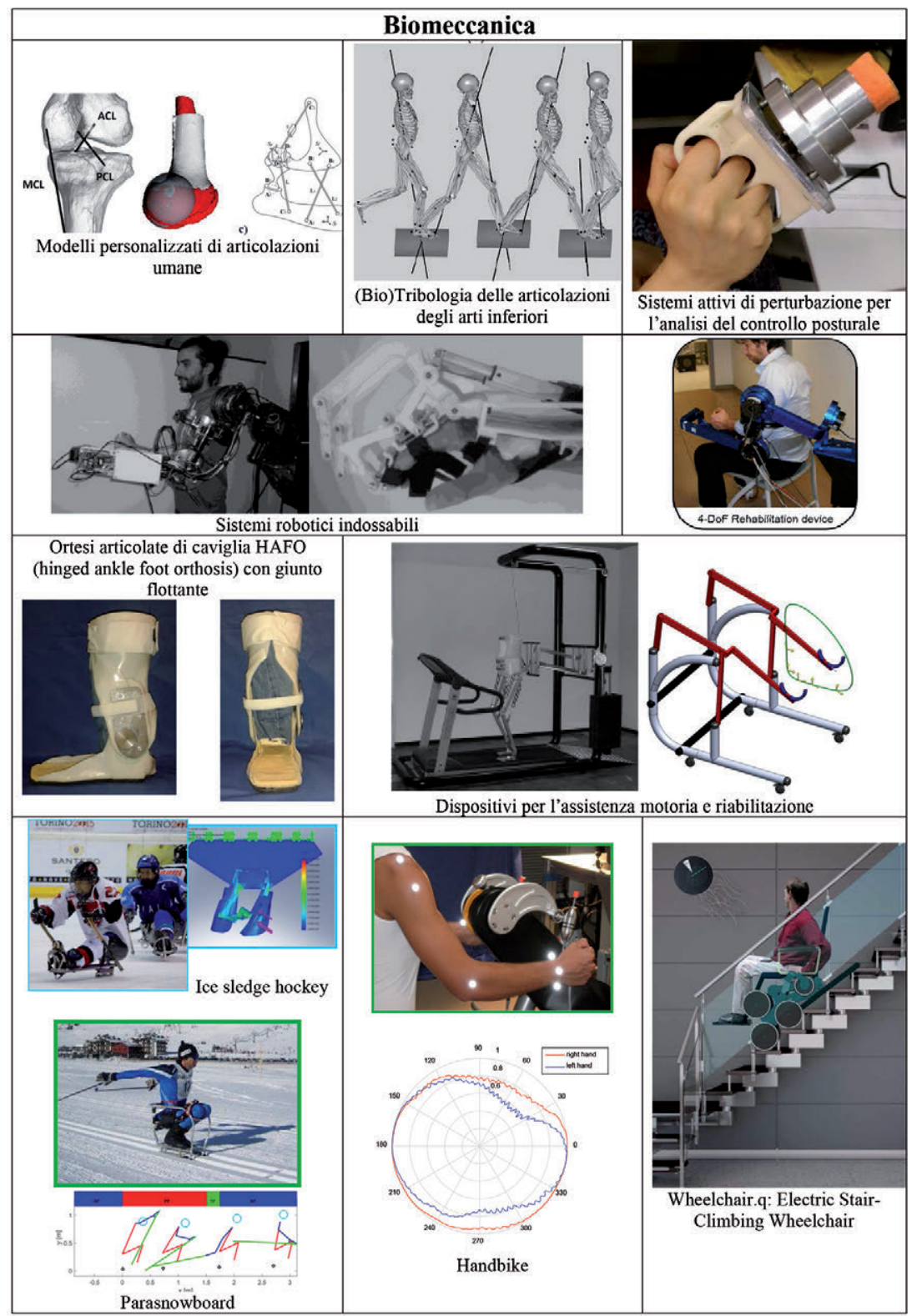

Fig. 4. Ricerche nel campo Biomeccanica. 


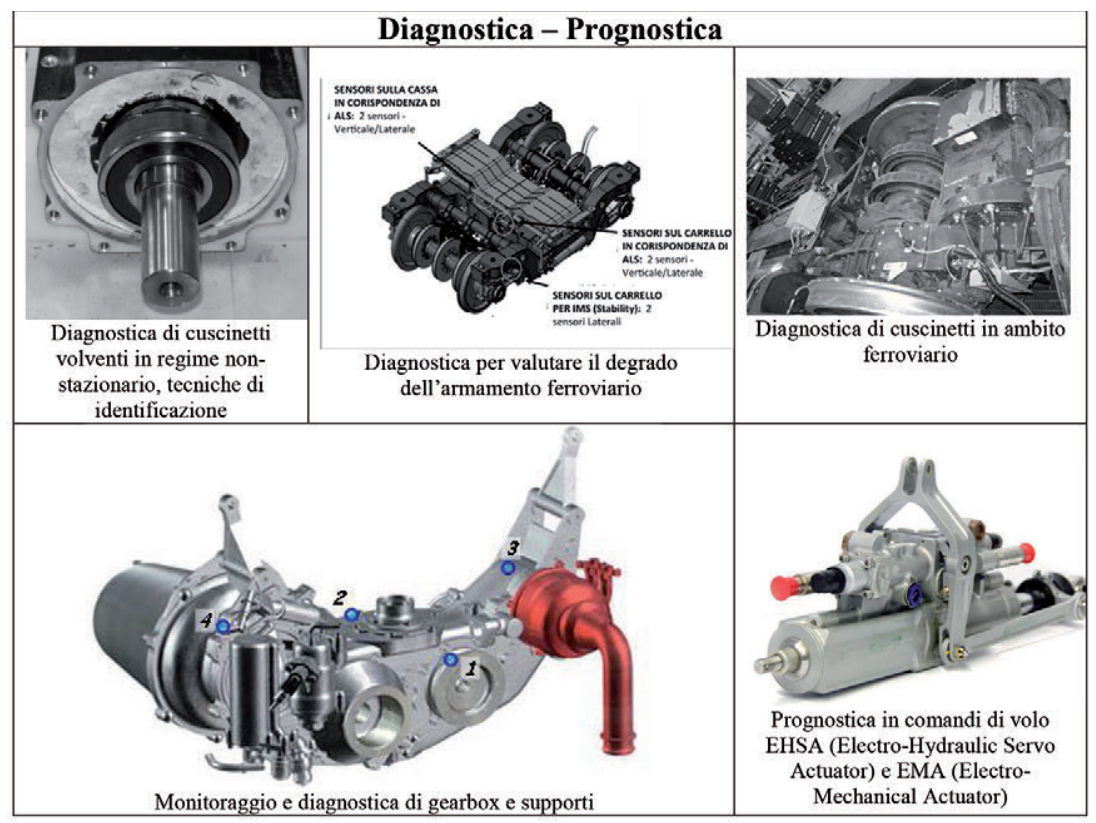

Fig. 5. Ricerche nel campo Diagnostica-Prognostica. 


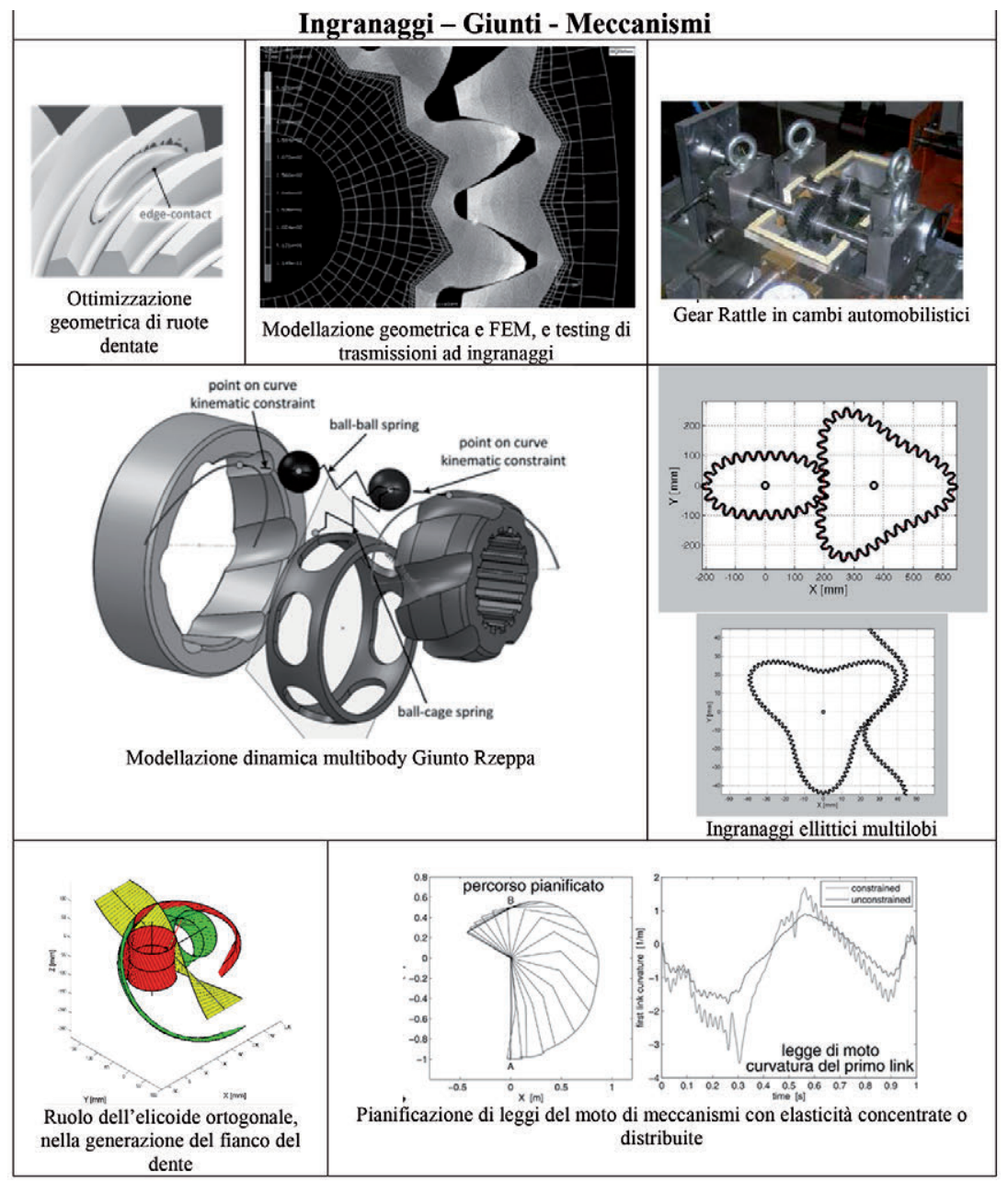

Fig. 6. Ricerche nel campo Ingranaggi-Giunti-Meccanismi. 


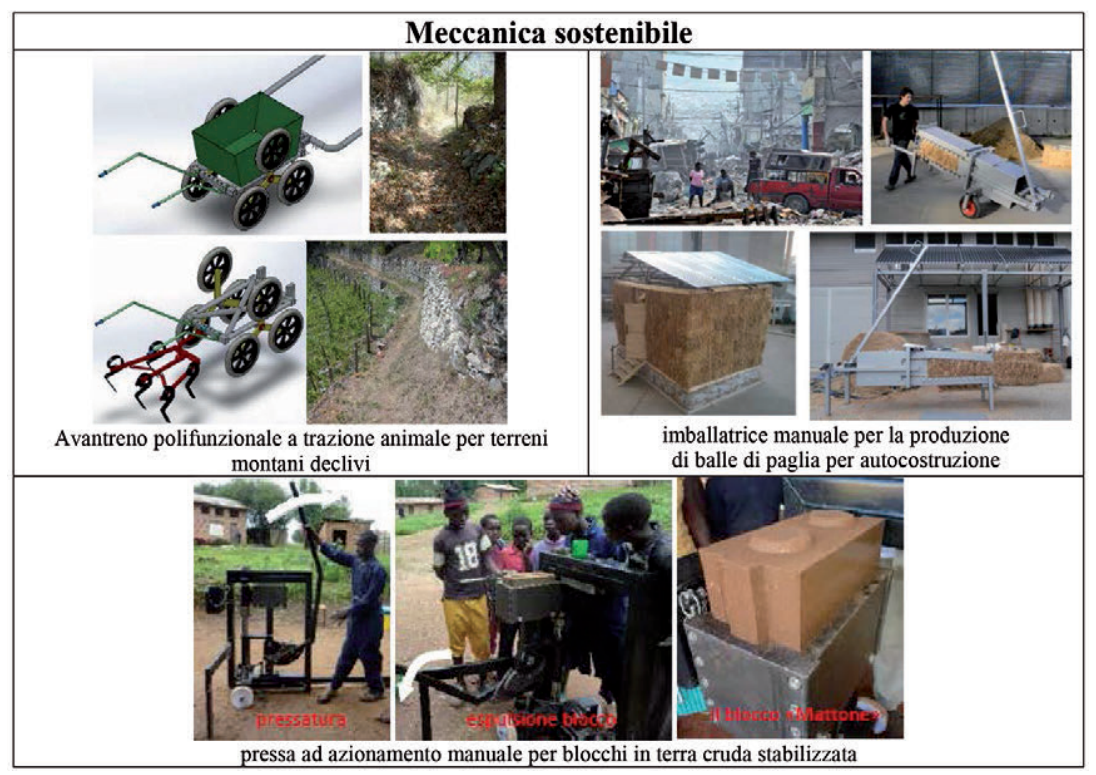

Fig. 7. Ricerche nel campo Meccanica sostenibile.

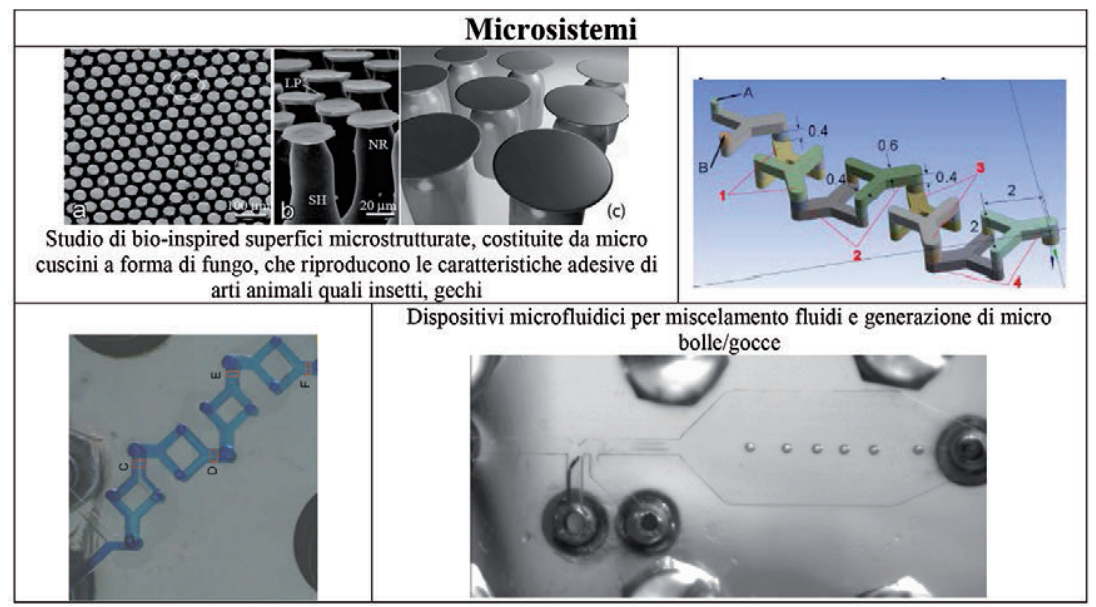

Fig. 8. Ricerche nel campo Microsistemi. 


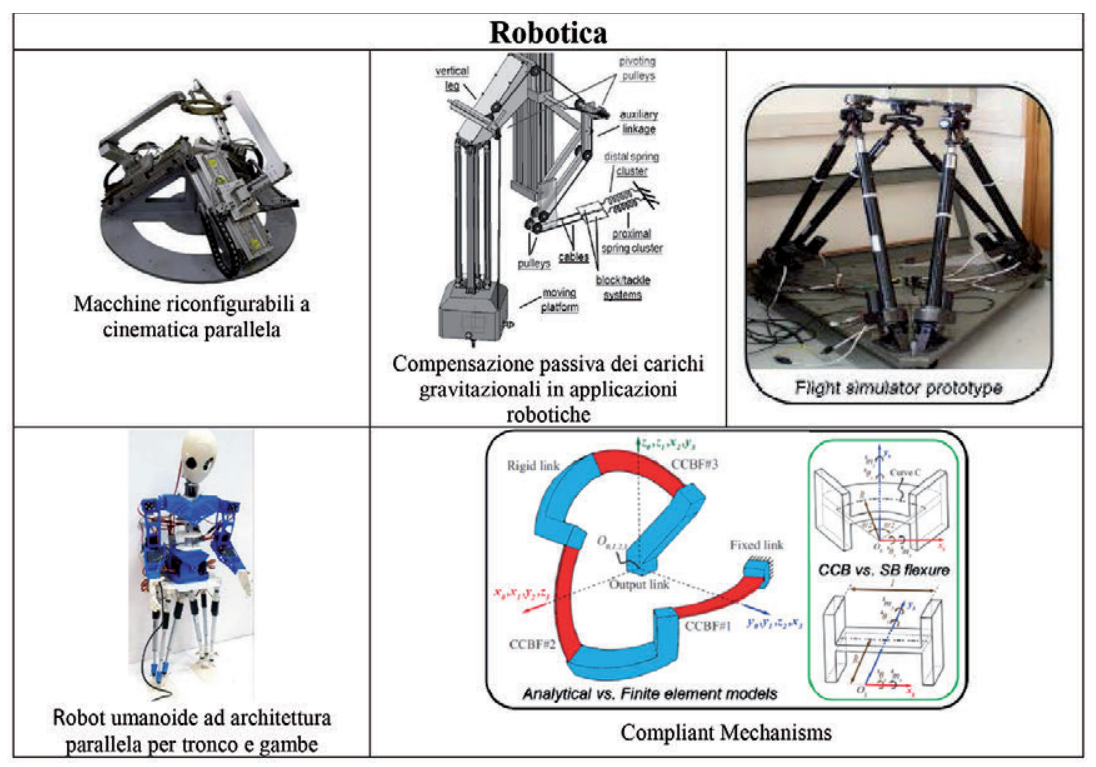

Fig. 9. Ricerche nel campo Robotica.

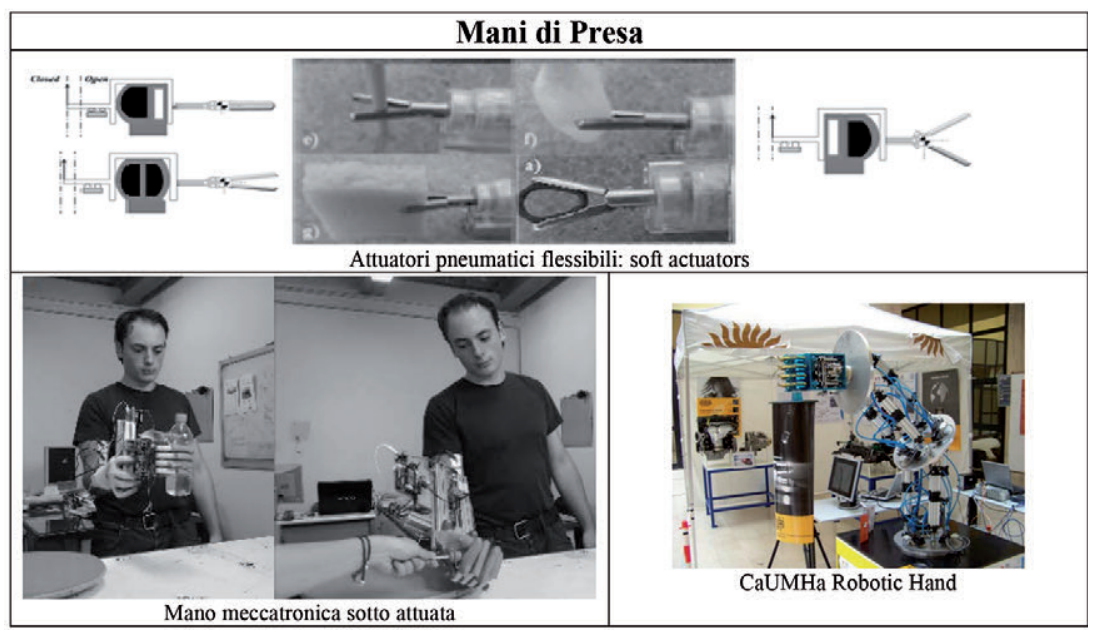

Fig. 10. Ricerche nel campo Mani di presa. 


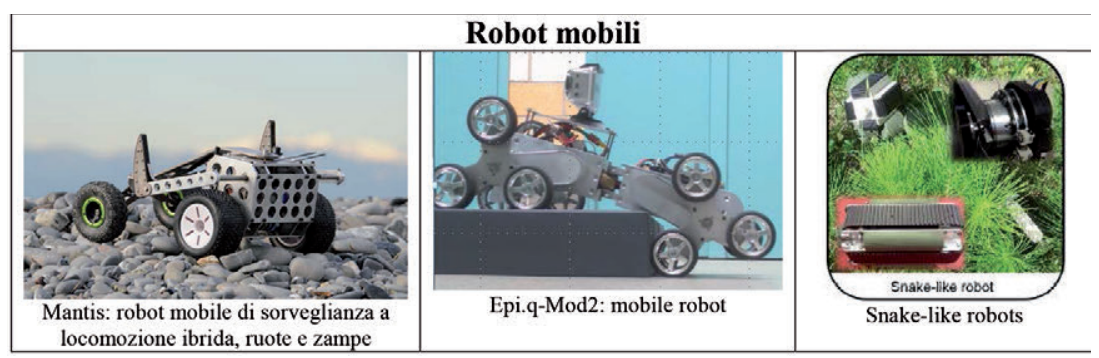

Fig. 11. Ricerche nel campo Robot mobili.

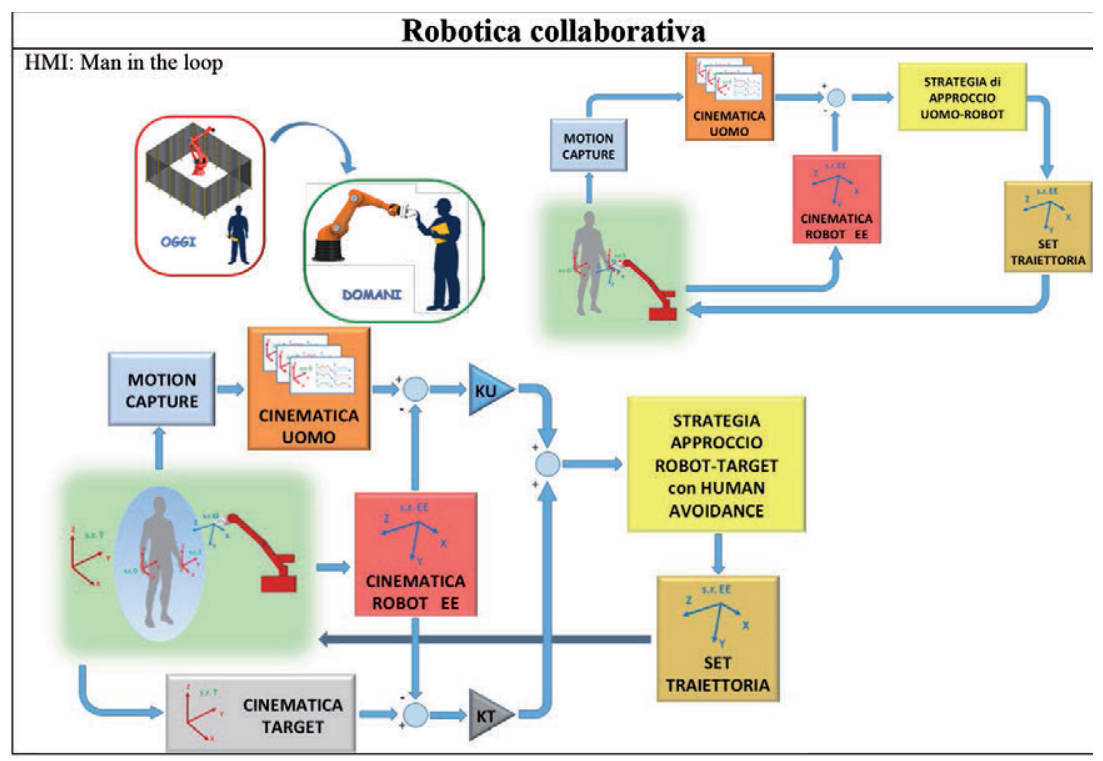

Fig. 12. Ricerche nel campo Robotica collaborativa. 


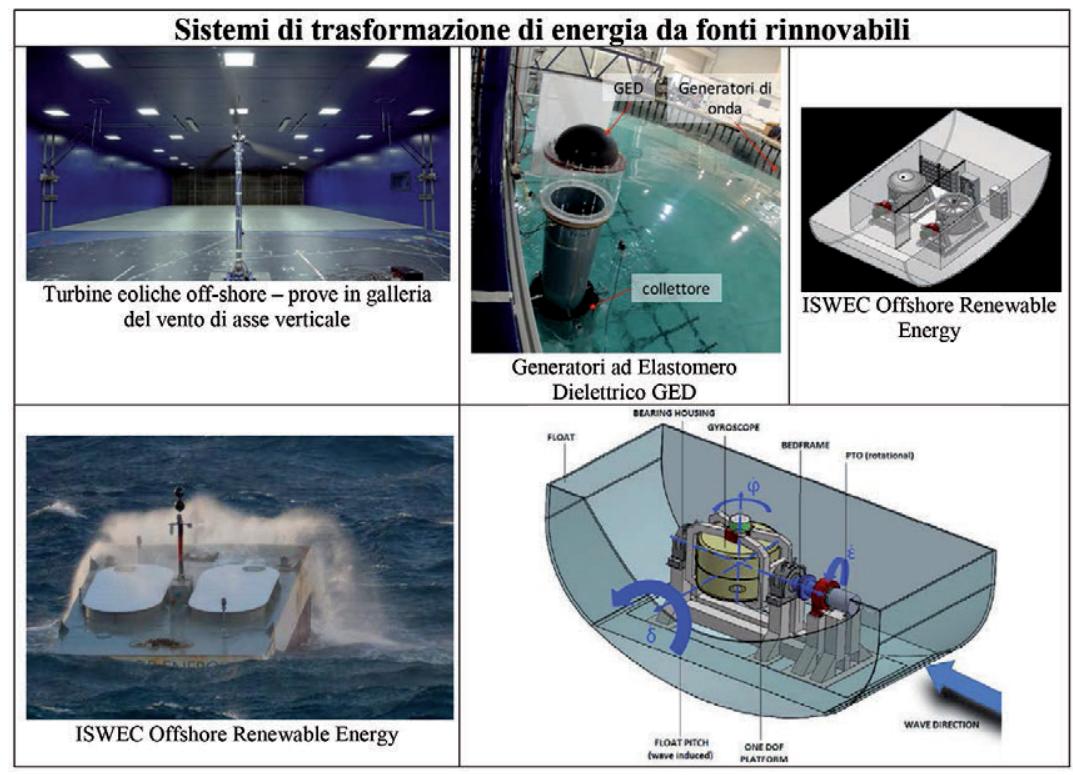

Fig. 13. Ricerche nel campo Sistemi di trasformazione di energie rinnovabili.

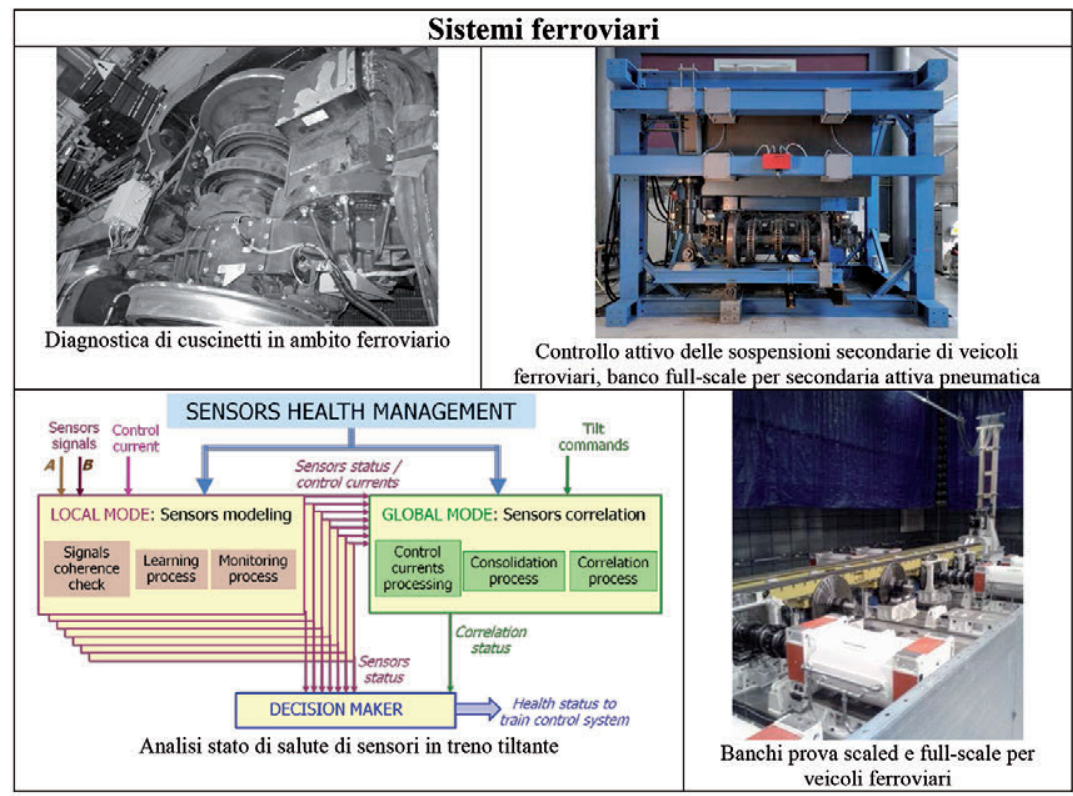

Fig. 14. Ricerche nel campo Sistemi Ferroviari. 




Fig. 15. Ricerche nel campo Sistemi Meccatronici. 


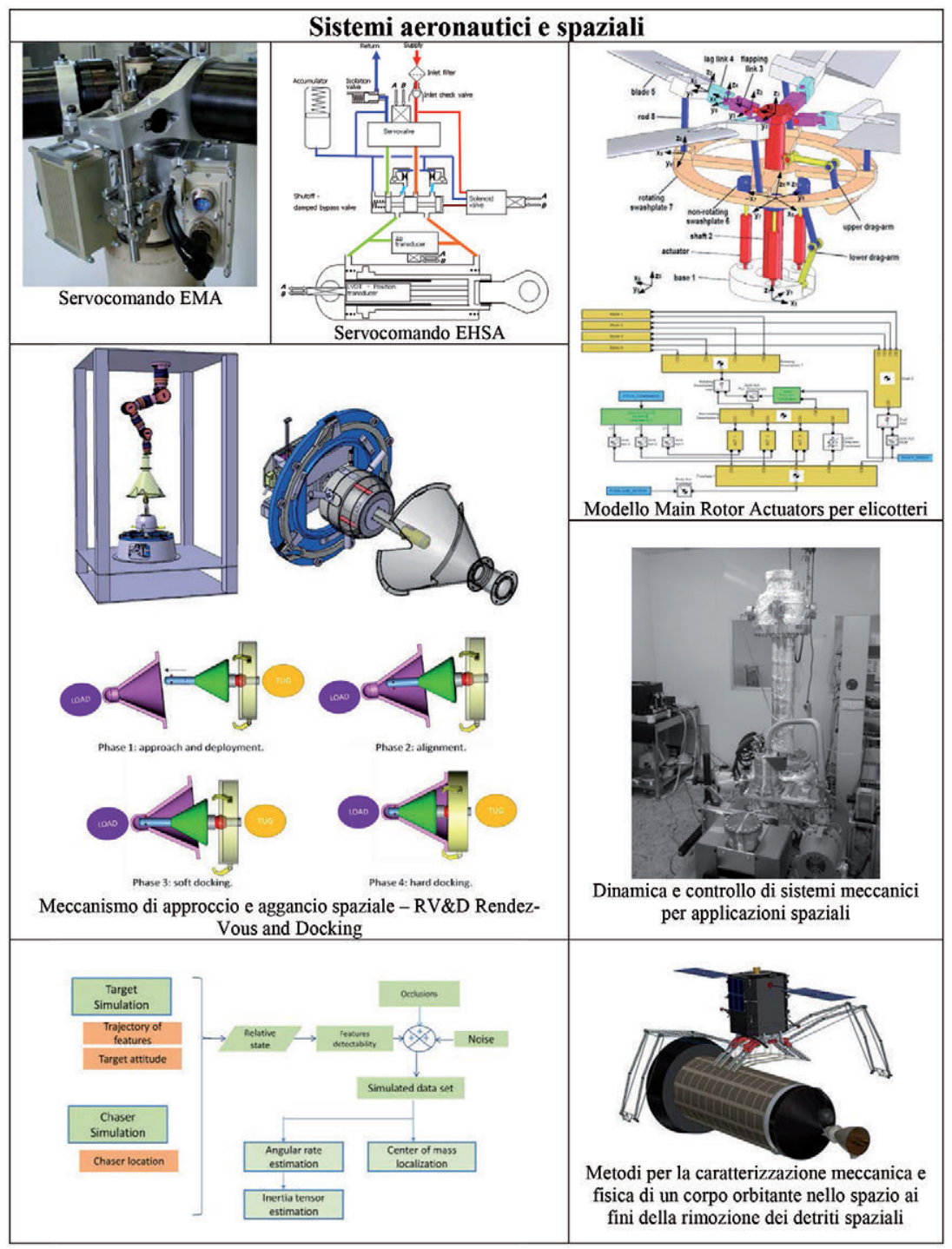

Fig. 16. Ricerche nel campo Sistemi Aeronautici e Spaziali. 


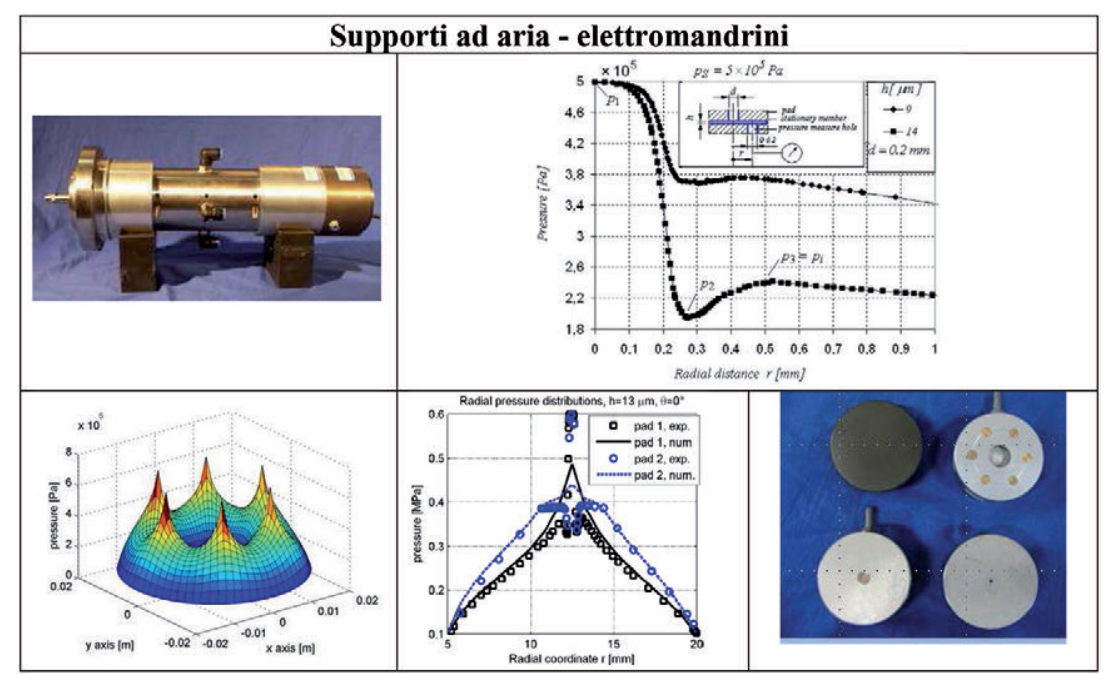

Fig. 17. Ricerche nel campo Supporti ad Aria e Elettromandrini.

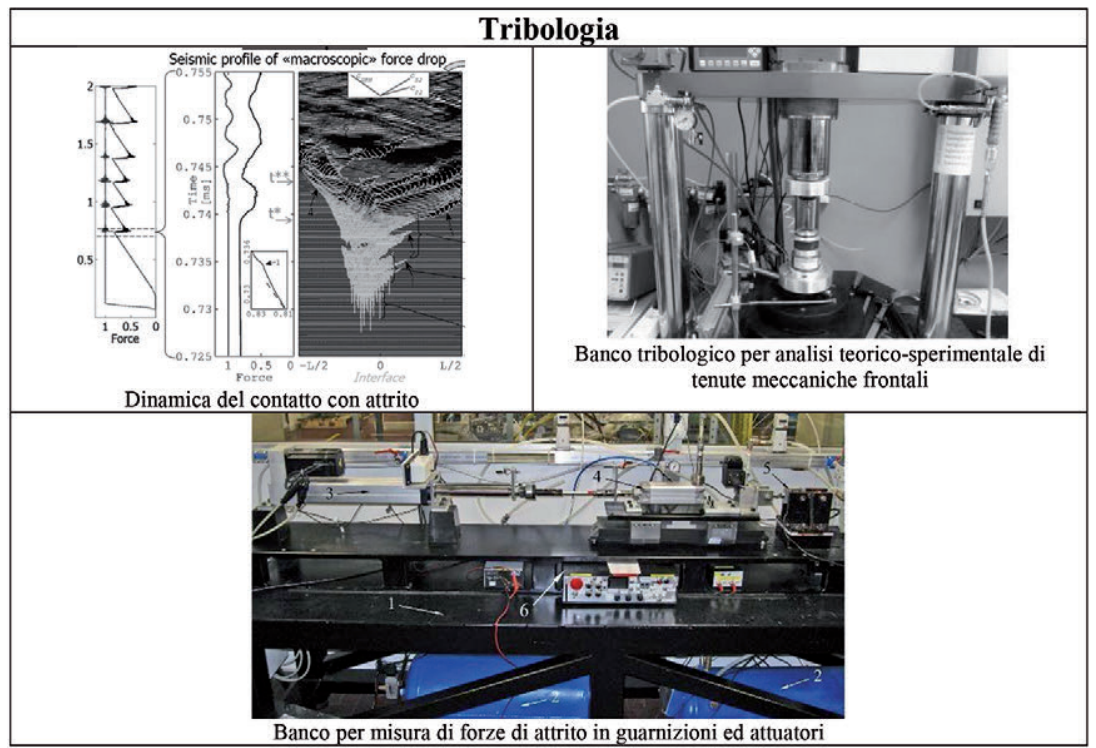

Fig. 18. Ricerche nel campo Tribologia. 




Fig. 19. Ricerche nel campo Identificazione Sistemi Vibranti. 


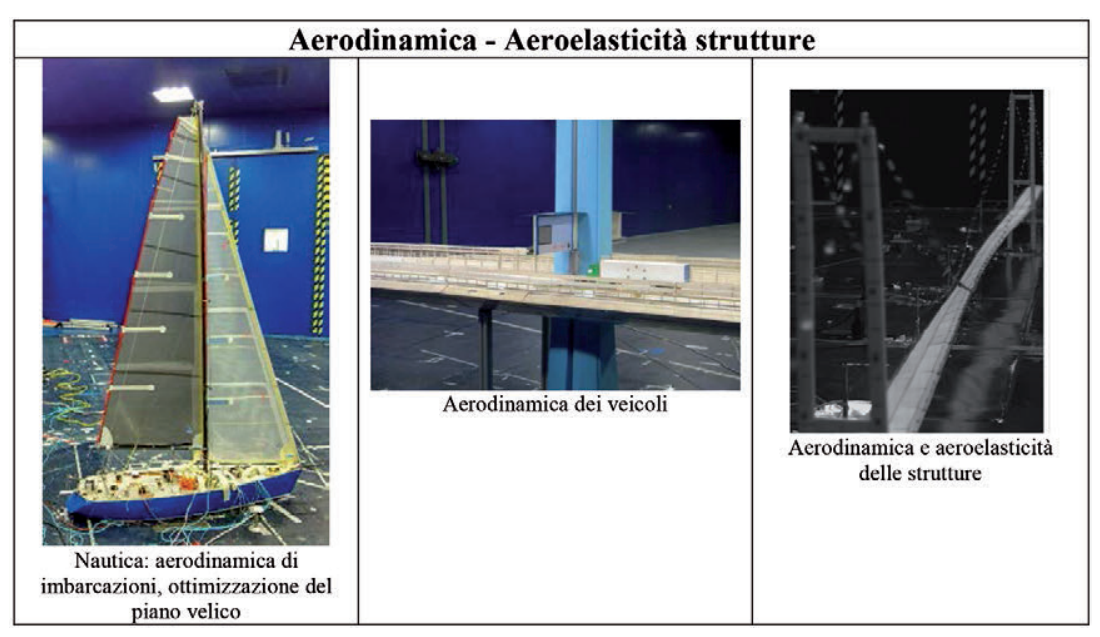

Fig. 20. Ricerche nel campo Aerodinamica - Aeroelasticità di strutture.

\section{NuOve Tendenze: LA PROGNOSTICA COME ESEMPiO DI INTEGRAZIONE DELLA MDM}

La manutenzione in molti domini applicativi costituisce una voce di costo molto elevata nella gestione di un sistema. Sicuramente questa assunzione è vera nel settore della aviazione, dove la manutenzione del velivolo è una delle voci più costose affrontate dalle compagnie aeree, e contribuisce in larga parte al costo finale del ciclo di vita dell'aeromobile.

La prognostica (PHM Prognostics and Health Management) è una disciplina scientifica rivolta all'individuazione dei precursori di un guasto, alla valutazione dello stato di salute e in definitiva alla determinazione della vita utile residua di un dato componente o sistema [7]. Tale disciplina è correlata con la manutenzione basata sulla condizione CBM (Condition Based Maintenance), avente come obiettivo quello di massimizzare la durata di esercizio di un dato apparato e al tempo stesso di ridurre al minimo i costi di funzionamento e di manutenzione. La prognostica in definitiva ha come risultato la riduzione dei costi di funzionamento e manutenzione e infine del costo del ciclo di vita di un prodotto. Inoltre, un efficace sistema prognostico può contribuire ad aumentare il livello di sicurezza del sistema in cui un dato equipaggiamento è installato. 
La percezione delle possibilità offerte dalla prognostica ha portato negli anni recenti allo sviluppo e alla conduzione di attività di ricerca sulla prognostica in diversi campi applicativi, fra i quali le strutture in genere (impianti nucleari, strutture aeronautiche, infrastrutture), i dispositivi elettronici e, nello specifico settore aerospaziale, le trasmissioni, i gruppi di ingranaggi, le palette di turbine di motori aeronautici, i sistemi idraulici e i relativi servocomandi e azionamenti. In questi ultimi campi, l'attività di ricerca e sviluppo è a stadi di sviluppo tecnologico ancora iniziale, TRL 2-3.

\subsection{Obiettivi e limiti della prognostica}

L'incremento di complessità dei sistemi, unito alla tendenza alla diminuzione del personale addetto alla manutenzione, presuppone lo sviluppo di schemi di manutenzione più intelligente che sostituiscano le precedenti e costose procedure di manutenzione programmata, genericamente superate, ma che garantiscano al tempo stesso la corretta funzionalità del sistema e la sua assoluta sicurezza. Il raggiungimento di questi obiettivi richiede lo sviluppo di sistemi capaci di sfruttare le informazioni disponibili dai sistemi, [8], [9], e di elaborarle in modo efficace al fine di: i) determinare lo stato di salute del sistema, ii) individuare i segnali precursori di eventuali guasti, ossia i difetti in progress, iii) prevederne l'evoluzione nel tempo, iv) valutare la vita utile residua prima che il difetto determini la perdita di funzionalità.

Lo sviluppo di algoritmi di prognostica applicati ad un determinato dispositivo potrà portare ai seguenti vantaggi:

- Riduzione della frequenza con la quale il dispositivo viene rimosso in seguito a falsi allarmi di guasto (no-fault found).

- Riduzione del numero di parti di ricambio nei magazzini poiché l'insorgenza di un guasto può essere scoperta in anticipo.

- Minor tempo speso per periodiche ispezioni poiché l'algoritmo di prognostica stabilisce se esiste un problema.

- Minor tempo speso per identificare un guasto, poiché questa funzione è svolta dall'algoritmo di prognostica.

- Riduzione del numero di incidenti connessi al malfunzionamento del dispositivo poiché l'algoritmo di prognostica individua i segnali precursori del guasto. 
Le limitazioni e le problematiche che un sistema di prognostica presenta possono essere così espressi:

- Si utilizzano in generale solo le informazioni trasmesse dai sensori già disponibili, senza dunque libera possibilità di inserire ulteriori trasduttori all'interno del sistema. Questa limitazione nell'incremento del numero di sensori e di conseguenza di informazioni utili per l'individuazione dell'innesco del difetto, legata da un lato alla necessità di non ridurre l'affidabilità del sistema sotto esame e dall'altra alla reale possibilità di implementazione di nuove configurazioni a costi ragionevoli su architetture già in servizio, è uno dei maggiori problemi che limita lo sviluppo di un sistema PHM.

- Sviluppare un algoritmo PHM che sia in grado di identificare tutte le possibili modalità di guasto non è una operazione conveniente né dal punto di vista economico né dal punto di vista del tempo impiegato per effettuare l'identificazione.

- I tipi di guasti che si verificano più comunemente in molte applicazioni sono già ben noti dall'esperienza, anche se non esistono modelli consolidati per tali modalità di guasto che possano essere presi come riferimento per la predizione della loro degradazione progressiva. Tipico è il caso dei sistemi di attuazione meccatronici, quali ad esempio i comandi di volo fly-by-wire elettroidraulici EHSA. Sebbene i modelli di crescita del guasto non siano ancora stati completamente validati, il loro approccio "fisico" al problema assicura che la crescita della degradazione sia descritta correttamente, consentendo l'implementazione di una prova virtuale mediante l'utilizzo di algoritmi di controllo della salute efficaci.

\subsection{Strumenti indispensabili per la prognostica}

Lo sviluppo di un sistema prognostico richiede, oltre che una ampia competenza circa le caratteristiche fisiche del sistema, anche la conoscenza delle degradazioni che possono svilupparsi nel sistema nelle diverse fasi di funzionamento. Solo per potere fare qualche esempio le degradazioni in un sistema meccatronico possono essere correlate a i) incremento delle azioni di attrito, per modifica delle superfici e/o delle caratteristiche tribologiche dei materiali; ii) variazioni di quote geometriche per effetto di usura; iii) incremento del gioco in coppie cinematiche e/o organi in movimento relativo, per variazione di rigidezza, usura; iv) incremento di trafi- 
lamenti interni, per usura meccanica degli organi di tenuta, per usura chimica, per presenza di debris nel fluido; v) degradazioni delle proprietà elettro-magnetiche di un motore, di un trasduttore, o di un dispositivo di interfaccia (torque motor, servosolenoide, ...).

L'interpretazione del fenomeno fisico associato alle cause della genesi delle degradazioni, e soprattutto la conoscenza dei processi di accrescimento dei difetti, trovano una possibile soluzione solo in una accurata conoscenza della fisica di base del fenomeno interessato. In altri termini per potere dare un contributo alla prognostica occorre svolgere approfondimenti nella ricerca di base, multifisica e fortemente supportata da tecniche analitiche/matematiche statistiche e probabilistiche accurate. Occorre quindi considerare che gran parte delle degradazioni e dei difetti che si determinano nella fase preliminare, ossia che si manifestano come precursori del guasto, con sistema ancora pienamente operativo, sono del tutto definite nelle tematiche della Meccanica delle Macchine, anzi, sollecitano e propongono nuovi filoni di ricerca e metodologie di sperimentazione, e non ultimo sempre maggiore integrazione, da un lato con le scienze matematiche, dall'altro con la reale applicazione sul campo.

E' molto importante, soprattutto in fase iniziale, definire quali siano le tipologie di degradazione per le quali risulta assolutamente conveniente sviluppare un algoritmo prognostico. Quest'ultime saranno quelle la cui probabilità che avvengano risulta molto alta e il cui accadimento è critico per il corretto funzionamento dell'intero sistema.

Occorre quindi ipotizzare e/o assumere una legge di crescita della degradazione efficacemente correlata con un approccio fisico legato al reale funzionamento del dispositivo/sistema, sfruttando per quanto possibile il risultato di ambienti virtuali di simulazione multifisica del sistema.

A tale proposito è indispensabile, almeno in fase di progetto, realizzare un modello high-fidelity del funzionamento statico e dinamico del sistema, in grado di simulare gli andamenti temporali delle grandezze fisiche principali, con una accuratezza sufficiente a stimare e predire anche le variazioni dei segnali derivanti da ridotte variazioni dei parametri, ossia derivanti da iniziali degradazioni, precursori di guasto. Il modello di simulazione può essere anche indispensabile per generare, laddove non disponibili in quanto non memorizzati in precedenza, una banca dati di difetti dello specifico sistema, sul quale potere progettare e ottimizzare opportunamente l'algoritmo di prognostica.

In definitiva è necessario validare l'ambiente di simulazione highfidelity con test sperimentali in condizioni di baseline, ossia di configu- 
razione standard del sistema, senza difetti, quindi tramite storie temporali acquisite o da precedenti test del sistema fisico montato nella sua configurazione operativa finale, o da test condotti su appositi banchi di prova sul quale il dispositivo in prova deve essere testato in condizioni il più possibile simili a quelle operative finali.

\subsection{Definizione e estrazione delle features o indici di salute}

La feature è una misura quantitativa di dati numerici o sperimentali, corrispondente a uno specifico segnale o a una elaborazione dello stesso (ad esempio gain/amplitude della risposta in frequenza, fase, corrente massima di un dispositivo elettromagnetico, ...), che esprime un indicatore, un indice di salute del sistema. Da tale misura si possono estrarre informazioni utili, in forma compatta, sulla base della fisica dei meccanismi di guasto e dell'esperienza sul campo.

Le features devono essere strettamente correlate alla degradazione, al fine di consentire una loro facile identificazione; inoltre sarebbe estremamente efficace se fossero ognuna particolarmente sensibile ad una singola degradazione, per consentire la loro rapida identificazione e quindi ridurre il tempo per la stima della vita utile residua, e dunque ridurre al massimo l'intervallo di tempo fra il riconoscimento del difetto e l'intervento di manutenzione. Nello stesso tempo questi indici devono essere robusti nei confronti di una eventuale variazione delle condizioni operative ed ambientali per evitare il più possibile falsi allarmi e aumentare in tal modo il livello di precisione dell'algoritmo PHM.

La determinazione delle features, in fase di validazione dell'algoritmo di identificazione dei fault e di predizione della vita utile residua, può essere effettuata mediante lo svolgimento di una serie di simulazioni con il modello multifisico high fidelity, nelle quali viene imposta una opportuna legge di input, una sequenza di comandi inviati in serie. Tale legge presenta andamenti temporali con contenuto in frequenza correlato con le features che si prevede di delineare. A titolo di esempio in Fig. 21 è riportata una sequenza temporale, ossia un set di input, impiegata per l'analisi delle features di comandi di volo elettroidraulici EHSA (Electro-Hydraulic Servo-Actuator) sviluppata in [10].

Nel caso dello stesso equipaggiamento EHSA, l'estrazione delle feature effettuata operando opportunamente sulle storie temporali di uscita, del tipo di Fig. 22, si esplica nella valutazione degli indici di salute mostrati nella Fig. 23, che descritto in [10] e [11]. 
La valutazione delle Features è rivolta a definire quanto esse siano buoni indicatori della presenza di eventuali degradazioni all'interno del dispositivo. L'obbiettivo è quello di correlare un determinato livello di degradazione con una corrispondente variazione dell'indice esaminato, ripetendo ad esempio la sequenza di Fig. 21 a intervalli di tempo successivi, che corrispondono a una certa ipotesi di crescita della degradazione. Sono disponibili numerose metriche che consentono la valutazione degli indici e che portano ad identificare e a classificare tutte quelle features che meglio rappresentano lo stato del sistema [7]. Fra le metriche si possono citare il Coefficiente di correlazione e la Distanza massima delle misure.

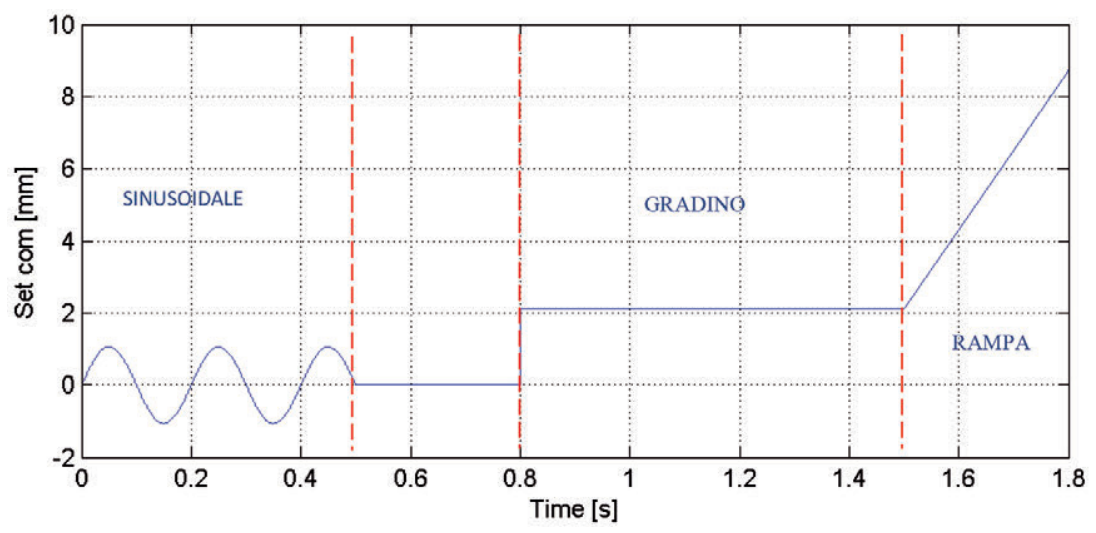

Fig. 21. Esempio di SET di comando pre/post volo per comandi di volo EHSA.

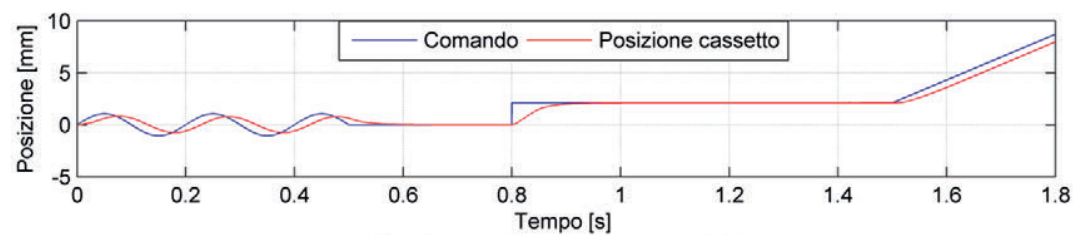

a) Set di comando e spostamento del cilindro

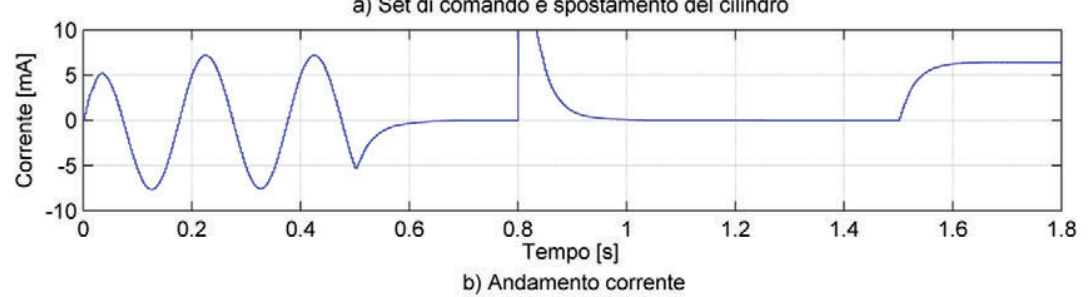

Fig. 22. Comando e risposta in test per comandi di volo EHSA. 


\begin{tabular}{|c|c|c|}
\hline $\begin{array}{c}\text { Tipo di comando al } \\
\text { servoattuatore }\end{array}$ & $\begin{array}{c}\text { Features di posizione } \\
\text { attuatore }\end{array}$ & $\begin{array}{c}\text { Features di corrente } \\
\text { servovalvola }\end{array}$ \\
\hline Sinusoidale & $\begin{array}{c}\text { Gain } \\
\text { Phase }\end{array}$ & $\begin{array}{c}\text { Current max } \\
\text { Current min } \\
\text { Current mean }\end{array}$ \\
\hline Step & Rise time & Time decrease \\
\hline Ramp & Steady state error & Current max ramp \\
\hline
\end{tabular}

Fig. 23. Indici di salute per test su comandi di volo EHSA.

Il coefficiente di correlazione tra due variabili è definito come:

$$
r_{d, F}=\frac{1}{N-1} \sum_{i=1}^{N}\left(\frac{\left(D_{i}-\mu_{D}\right)\left(F_{i}-\mu_{F}\right)}{\sigma_{D} \cdot \sigma_{F}}\right)
$$

essendo $\mathrm{F}$ il valore della feature, $\mathrm{D}$ quello della degradazione per la quale si vuole studiare la correlazione della feature, $\mu$ e $\sigma$ rispettivamente la media e la deviazione standard delle due variabili, $\mathrm{N}$ è il numero di campioni. La condizione ottimale è quella di ottenere features che presentino un grado di correlazione elevato nei confronti di tutte le degradazioni in esame, in modo da ridurre al minimo la possibilità di falsi allarmi.

La distanza è definita come la variazione percentuale tra il valore nominale della feature, in assenza di degradazione e il valore di quest'ultima in corrispondenza della massima degradazione

$$
\text { Dist }=\left|\frac{\left(\mu_{F_{\text {nom }}}-\mu_{F}\right)}{\mu_{F_{\text {nom }}}}\right|
$$

Un valore Dist molto elevato implica una maggiore possibilità di identificazione del guasto selezionato senza la presenza di falsi allarmi, dal momento che le piccole variazioni introdotte dai disturbi diventano trascurabili.

\subsection{Identificazione del guasto}

Differenti sono le modalità di identificazione del guasto, fra le quali: i) l'utilizzo di opportune bande di tolleranza (Alarm bounds), per esempio le bande di Bollinger, ii) la distribuzione dell'errore relativo assoluto rispetto al valore nominale.

L'algoritmo per l'identificazione del difetto basato sulla distribuzio- 
ne dell'errore è visualizzato in Fig. 24, ancora per un servocomando EHSA. La distribuzione di riferimento viene acquisita utilizzando una finestra di 50 acquisizioni, effettuate quando l'equipaggiamento è in condizioni nominali e privo di degradazioni. Questa tecnica consente all'algoritmo di auto-adattarsi alle caratteristiche proprie di ciascun dispositivo derivanti da possibili dispersioni del processo di produzione. La distribuzione di riferimento può essere acquisita quando l'attuatore viene installato per la prima volta sul velivolo o dopo aver effettuato la manutenzione del componente. La distribuzione corrente dell'errore relativo viene acquisita all'interno di una finestra mobile di 50 acquisizioni con overlap di 49. Le due distribuzioni vengono quindi confrontate verificandone il ricoprimento. Quando viene identificata una degradazione viene inviato un messaggio di avvenuta identificazione e da quel momento viene attivata la routine di classificazione del difetto e di stima della vita utile residua.

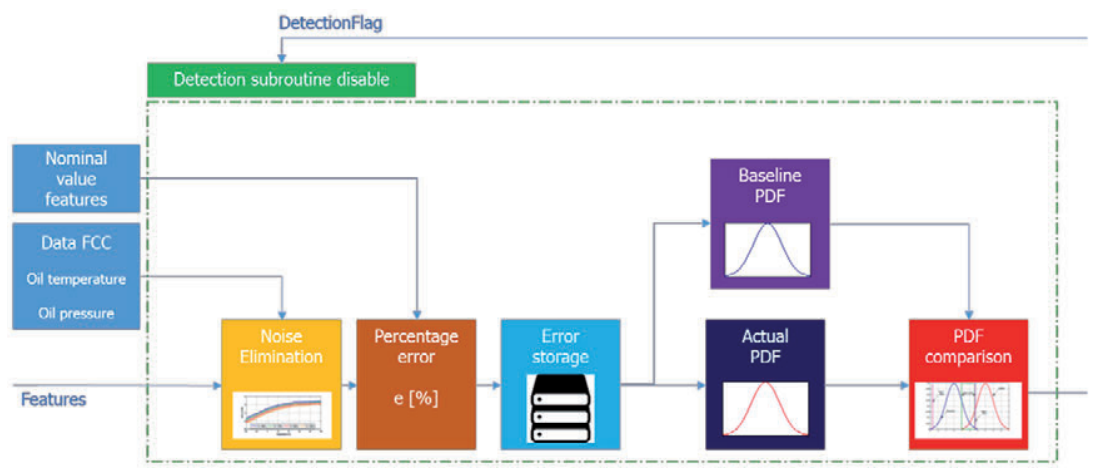

Fig. 24. Identificazione del difetto basato sulla distribuzione dell' errore.

La presenza di un difetto viene riconosciuta quando il ricoprimento delle due distribuzioni PDF (funzioni densità di probabilità) è minore di un certo valore limite, contemporaneamente viene indicato il livello di confidenza di identificazione del difetto. Vengono definiti il valore limite e il livello di confidenza necessario per l'identificazione della degradazione, definendo il type I e il type II error, ovvero i parametri per l'identificazione del difetto:

- Il type I error coincide con la probabilità di riscontro di falsi allarmi.

- Il type II error coincide con il livello di confidenza con il quale un difetto viene identificato. 
Una rappresentazione grafica della procedura di confronto della distribuzione di riferimento e della distribuzione corrente è mostrata in Fig. 25, dove sono altresì mostrati il limite di identificazione del difetto e l'intervallo di confidenza di identificazione.

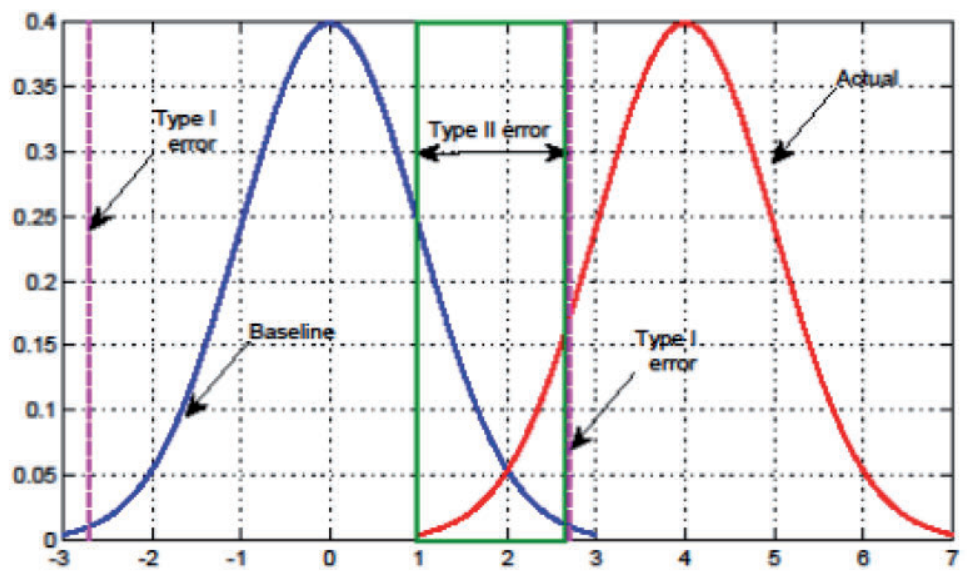

Fig. 25. Confronto distribuzioni errore relativo e criteri di identificazione difetto. Fig. 26.

I valori scelti per il type I error e type II error sono definiti nella

\begin{tabular}{|l|l|}
\hline type I error \% & $5 \%$ \\
\hline type II error \% & $95 \%$ \\
\hline Moving window N. Sample & 50 \\
\hline N. bin for PDF approximation & 30 \\
\hline
\end{tabular}

Fig. 26. Definizione dei parametri per l'identificazione del difetto.

Un esempio di interfaccia grafica della procedura di identificazione del difetto in servocomandi EHSA è mostrato in Fig. 27. In alto è mostrata l'evoluzione del difetto in funzione delle ore di volo, impostata nel modello di simulazione e non nota all'algoritmo di PHM. Nella parte centrale della figura è mostrata l'evoluzione della confidenza di identificazione nel tempo. Nell'esempio riportato l'identificazione del guasto avviene al tempo 554 ore di volo, quando il livello 
di confidenza supera il limite del $95 \%$ definito in base al type II error. In basso viene mostrata la distribuzione di riferimento e la distribuzione corrente all'istante in cui il difetto è stato riconosciuto.

\section{At time 554.584:}

Type $\mathrm{I}=\mathbf{2 5} \%$ Confidence $=\mathbf{9 6} \%($ Estimate degradation $=\mathbf{1 6 . 4 3 \%})$
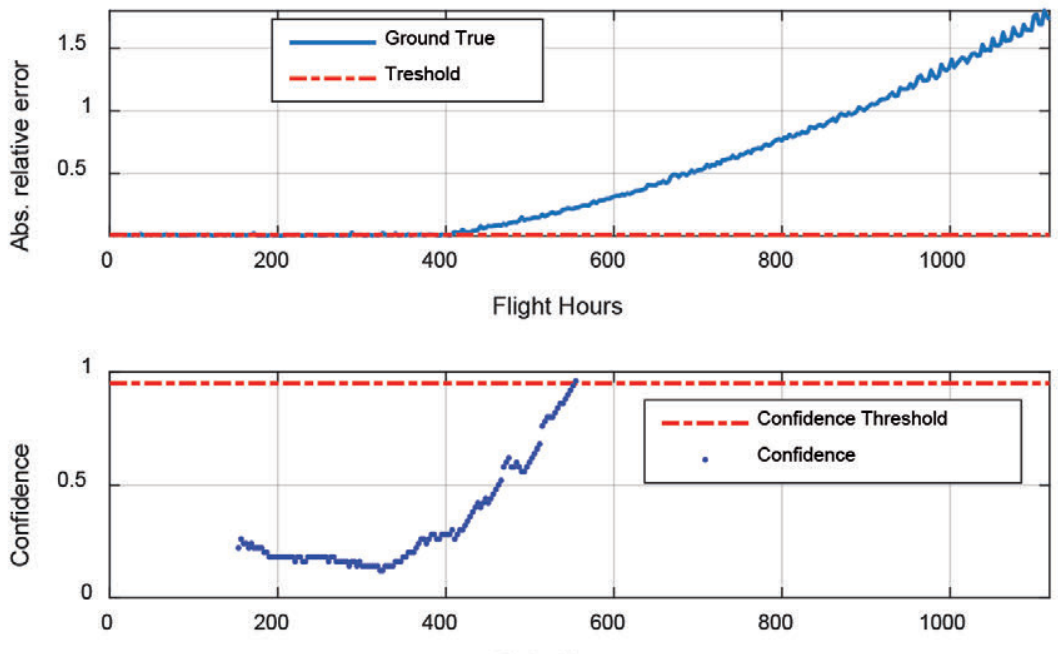

Flight Hours

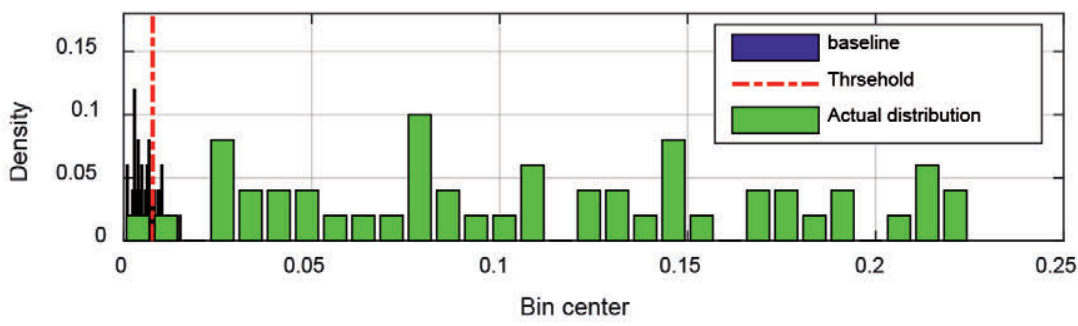

Fig. 27. Grafica della procedura di identificazione del difetto.

Tale risultato è orientato agli equipaggiamenti sui velivoli, ma i risultati ottenuti saranno utilizzabili anche nelle applicazioni su altre piattaforme (navi, veicoli terrestri). Inoltre, le metodologie sviluppate per realizzare algoritmi efficaci di prognostica per i servocomandi idraulici possono essere utilizzate ad esempio per lo sviluppo di siste- 
mi di prognostica dell'impianto idraulico nel suo complesso, o di componenti significativi, quali pompe e gruppi filtri.

\subsection{Stima della vita utile residua}

L'errore relativo calcolato dalle routine precedenti viene utilizzato per stimare la vita utile residua RUL (Remaining Useful Life) del servosistema. La routine di stima della vita utile residua viene attivata quando viene riscontrata la presenza di una degradazione. L'evoluzione nel tempo dell'errore relativo viene predetta mediante l'utilizzo del Particle Filter [7] e [11]. L'algoritmo del Particle Filter permette di stimare l'evoluzione a lungo termine della distribuzione di probabilità di tale parametro, detto anche variabile di stato. La distribuzione di probabilità della variabile di stato è approssimata da una distribuzione casuale di particelle. La predizione inizia da una distribuzione di probabilità ipotizzata a priori e procede stimando la distribuzione di probabilità ad ogni istante temporale. La varianza della stima della distribuzione di probabilità tende ad aumentare nel tempo, come accade normalmente in ogni processo di proiezione di uno stato in istanti futuri. Le acquisizioni esterne dello stato del sistema, acquisite ad istanti temporali diversi, sono utilizzate per correggere la stima dello stato e la relativa varianza, diventando la nuova condizione iniziale del processo di stima. La stima dell' evoluzione della variabile di stato avviene sfruttando un modello non lineare, che rappresenta la legge di degradazione del componente.

La stima della vita utile residua (Fig. 28) consiste in tre passaggi sequenziali:

- Filtraggio dell'errore con un filtro a media mobile

- Interpolazione del modello mediante filtro ricorsivo per minimi quadrati (RLS - Recursive Least Squares)

- Stima dell'evoluzione del parametro tramite il Particle Filter

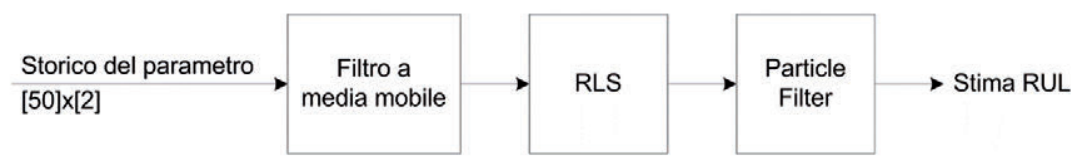

Fig. 28. Grafica della procedura di identificazione del difetto. 
In Fig. 29 è mostrato, un esempio di andamento temporale della RUL stimata e reale, con limite di accuratezza del 20\%. L'andamento ipotizzato della degradazione, imposto arbitrariamente, è lineare con inizio della propagazione al tempo di 40 ore e raggiungimento del limite di failure a 300 ore, corrispondente al 100\% di degradazione presente. In Fig. 29 l'asse temporale ha origine nell'istante di prima propagazione del difetto, mentre la stima della RUL viene effettuata dopo un tempo di circa 45 ore dall'inizio della crescita del difetto, ossia dopo il tempo che corrisponde al ritardo necessario per l'identificazione della degradazione da parte dell'algoritmo di diagnostica, e alla conseguente crescita della confidenza al $95 \%$.



Fig. 29. RUL per trafilamenti interni attuatore EHSA.

\section{CONCLUSIONI}

Il testo ha descritto le tematiche proprie della Meccanica delle Macchine, evidenziando i filoni di ricerca in atto nelle diverse sedi accademiche degli Atenei italiani.

L'attualità della Meccanica delle Macchine risulta evidente, sia 
per le tecnologie abilitanti che essa tocca, sia per quelle emergenti rivolte sia a fornire un miglioramento dei servizi nelle interazioni uomomacchina, sia a interagire con il settore delle energie alternative e rinnovabili, sia a evidenziare risvolti di tipo prettamente competitivo che vengono offerti alla innovazione dei prodotti e alla loro migliore collocazione sul rispettivo mercato.

In riferimento ad una di queste tecnologie emergenti, la prognostica, si è evidenziato che l'interpretazione del fenomeno fisico associato alle cause della genesi delle degradazioni, e soprattutto la conoscenza dei processi di accrescimento dei difetti, trovano una possibile soluzione solo attraverso una accurata conoscenza della fisica di base del fenomeno interessato. In altri termini per potere dare un contributo alla prognostica occorre svolgere approfondimenti nella ricerca di base, multifisica, e fortemente supportata da tecniche analitiche/matematiche statistiche e probabilistiche accurate. Occorre infine considerare che gran parte delle degradazioni e dei difetti che si determinano nella fase preliminare, ossia che si manifestano come precursori del guasto, con sistema ancora pienamente operativo, sono del tutto incluse nelle tematiche della Meccanica delle Macchine, anzi, sollecitano e propongono nuovi filoni di ricerca e metodologie di sperimentazione e non ultimo sempre una maggiore integrazione, da un lato con le scienze matematiche e dell'ICT, dall'altro con le reali applicazioni sul campo.

Risulta in definitiva che il mantenimento del ruolo della MdM, del tutto centrale nella interpretazione dei fenomeni fisici indispensabili alla innovazione dei prodotti e relativi processi, non può che determinare un beneficio per l'aggiornamento dei percorsi formativi e dei programmi dei corsi di insegnamento che ricadono nel Settore Scientifico Disciplinare della Meccanica Applicata alle Macchine.

\section{BIBLIOGRAFIA}

1. Jacazio G., Sorli M., Ferrara D., Smart force control system for real time loading of primary flight control actuators.5th Int. Conf. on Recent Advances in Aerospace Actuation Systems and Components. pp. 143-151. Toulouse, France, June 13-14, (2012).

2. Bertucci A., Jacazio G., Mornacchi A., Sorli M., A real time controlled test rig for high bandwidth force control. 8th FPNI Ph.D Symposium on Fluid Power. Lappeenranta, Finland, June 11-13, DOI 10.1115/FPNI2014-7813. (2014). 
3. Bertucci A., Mornacchi A., Jacazio G., Sorli M.. A Force Control Test Rig for the Dynamic Characterization of Helicopter Primary Flight Control Systems, Procedia Engineering, Volume 106, Pages 71-82, doi:10.1016/j.proeng.2015.06.010, ISSN 18777058 (2015).

4. Koetsier T., Kerle H., Yan H.S., The History of Mechanism and Machine Science (HMMS) and IFToMM's Permanent Commission for HMMS, Technology Developments: the Role of Mechanism and Machine Science and IFToMM, DOI 10.1007/978-94-007-1300-0_6, Springer, pp. 77-93. (2011).

5. Ceccarelli M., Activity and Trends in MMS from IFToMM Community, Technology Developments: the Role of Mechanism and Machine Science and IFToMM, DOI 10.1007/978-94-007-1300-0_1, Springer, pp. 3-24. (2011).

6. Autori Vari, Raccolta delle presentazioni delle attività di ricerca, Incontro GMA, Università degli Studi di Napoli Federico II, Napoli 21-22 luglio 2016, (2016).

7. Vachtsevanos G.J., Lewis F.L., Roemer M., Hess A., Wu B. Intelligent Fault Diagnosis and Prognosis for Engineering Systems, NY, John Wiley E Sons (2006).

8. Jacazio G., Bolognese D., Ferrara D., Sorli, Health management system for the pantographs of tilting trains, In: Proceedings of the First European Conference of the Prognostics and Health Management Society, PHM Society, Dresden 3-5 Luglio 2012, pp. 12, (2012), ISBN:9781936263042

9. Mornacchi A., Vachtsevanos G.J., Jacazio G., Prognostics and Health management of an Electro-Hydraulic Servo-Actuator. Annual conference of the Prognostics and Heath Management Society pp. 1-12, (2015).

10. G. Jacazio, A. Mornacchi, M. Sorli: - "Development of a Prognostics and Health Management System for ElectroHydraulic Servoactuators of Primary Flight Controls"- Proceedings of the 5th International Workshop on Aircraft Systems Technologies, Hamburg, 24-25 February, 2015, pagg. 13-22.

11. Mornacchi A., Design and Development of Prognostic and Health Management System for Fly-by-Wire Primary Flight Control Electrohydraulic Servoactuators, PHD Thesis, Politecnico di Torino, Italy, (2016). 